\title{
Automated Segmentation for Quantitative Analysis of Breast Thermograms
}

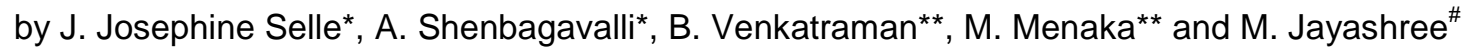 \\ * National Engineering College, Kovilpatti, Tamil Nadu, India, josieselle@gmail.com \\ **RSEG, Indira Gandhi Centre for Atomic Research, Kalpakkam, Tamil Nadu, India, menaka@igcar.gov.in \\ \# Department of Atomic Energy Hospital, Kalpakkam, Tamil Nadu, India, jayasree@igcar.gov.in
}

\begin{abstract}
Thermography in breast cancer detection presents promising diagnosis when used as a first line screening tool in adjunct to mammography. A thermogram consists of various regions such as patient's arms, chest and breasts. Segmentation of the breast region is an important step in classification of normal and abnormal thermogram. This paper deals with image pre-processing using inpaint algorithm and automated segmentation of breast regions using anisotropic diffusion filter along with level-set evolution without reinitialization. Three types of breast thermograms which include one normal and two abnormal were considered for this study. The segmented results were tested using performance measures and $98 \%$ of accuracy is obtained.
\end{abstract}

\section{Introduction}

There is a drastic increase in the mortality rates of women globally due to breast cancer. The World Health Organization (WHO) reports that breast cancer has become one of the deadliest as it overtakes the first place of cervical cancer. The analysis shows that 19.3 million new cases are to be predicted in the year 2025 which is much higher when compared to 2012 [1]. There is a need for early detection, and various modalities have emerged to diagnose the cancer. These techniques are either based on light (optical) or sound (ultrasound) or heat (thermography) or $\mathrm{X}$ rays (mammography).

Early papers on Infrared Imaging or Thermography present relationship between skin temperature and the breast [2-3]. In 1963 breast surgeon Dr. Ray Lawson's publication [4] stated that the metabolic activity and vascular circulation in both precancerous tissue and the area surrounding the breast developing a tumor is always higher than in normal breast tissue. This process frequently increases the regional surface temperature of the breast which can be detected by Infrared Imaging. Unlike other modalities that detect morphological changes, thermography finds the development of tumor before its onset [5-6]. It is painless, non-intrusive and the very important advantage is that it can be used on young women even with denser tissues which otherwise poses difficulty while viewing in x-ray mammography. Moreover, thermography is very useful in detecting histological pathologies in pregnant and lactating women as they need not face any ionizing radiation.

Digital Infrared Thermal Imaging (DITI) camera is used to obtain infrared images and these images are called as thermogram. The camera converts the infrared radiation emitted from the skin surface into electrical impulses that are visualized in color on a monitor. This visual image graphically maps the body temperature and the spectrum of colors indicates an increase or decrease in the amount of infrared radiation being emitted from the body surface. Since there is a high degree of thermal symmetry in the normal body, even slight asymmetries can be easily identified. The quality of these thermograms is poor when a radiologist directly views it. Hence, image processing techniques are applied for using IR as screening tool as well as for improving the diagnosis. In recent papers, special attention has been given to detect the tumors at early stages as it is a very important early sign of pathology.

This paper presents image processing methods and techniques applied on raw thermograms. For a successive interpretation, the region of interest must be clearly specific to process on a desired portion of the breast in order to analyze the defected pathology. The subsequent sections describe about label removing through inpaint algorithm, anisotropic diffusion filter for image enhancement and level set evolution for segmentation. Following that, experimental results and performance evaluation measures are presented in the later sections.

\section{Patients and methods}

\subsection{Patient preparation and imaging}

There is no such universally accepted acquisition protocol for breast thermal imaging. Hence, clinics and hospitals in other countries assumed different protocols and patient positioning while imaging. The setup of infrared imaging is shown in Figure 1. The images obtained in this paper, followed the procedure that is used in [7] where women were positioned static and images in five postures were captured: (i) frontal, (ii) right semi-oblique, (iii) left semi-oblique, (iv) right oblique and (v) left oblique. Every patient was asked to fill a breast health history form inquiring their age, full term of pregnancy, menstrual period and other health issues if any. A stable condition at $22^{\circ} \mathrm{C}$ was maintained in the imaging room. The patients were asked to undress up to waist so that their body temperature is free of sweat and equilibrates with the ambience. The camera was fixed at a distance of $2 \mathrm{~m}$ from the patient. All the patients were made to raise their arms above their head while taking images in different angles [8]. The patients were made to stand in five views as shown in Figure 2. 


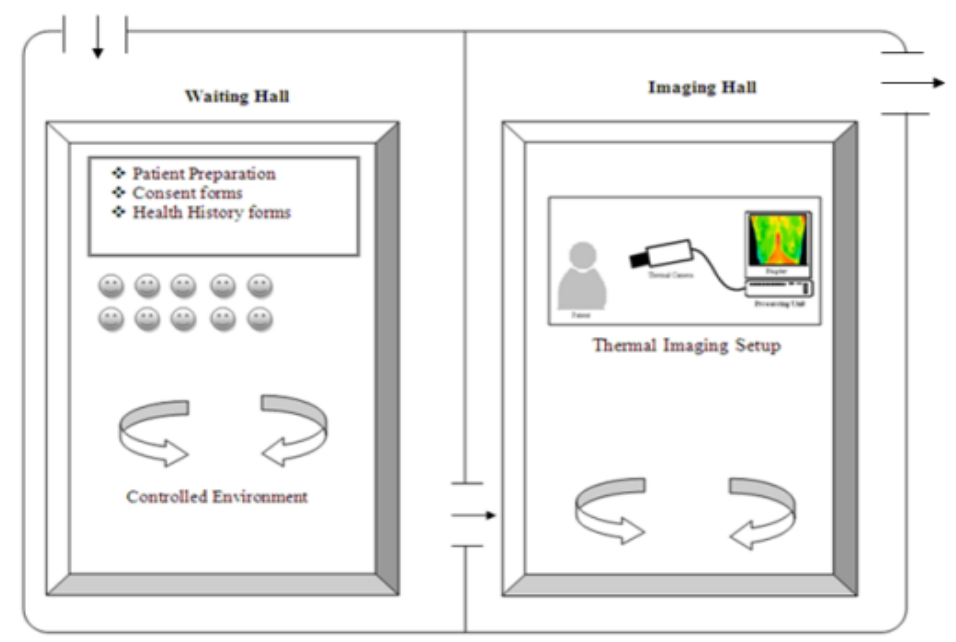

Figure 1: General layout for thermal imaging environment

\subsection{Thermographic camera}

The thermal camera used was FLIR T400. This new generation camera contains an uncooled Focal Plane Array (FPA) micro bolometer detector. The pixel resolution of the thermogram obtained is $320 \times 240$. The temperature range lies between $-20^{\circ} \mathrm{C}$ and $120^{\circ} \mathrm{C}$, whereas spectral range is 7.5 to $13 \mu \mathrm{m}$. The minimum detectable temperature difference is $0.05^{\circ} \mathrm{C}$ at $30^{\circ} \mathrm{C}$ normal mode. This camera is accompanied by Therma Report software which can be installed in the PC. The software supports thermal analysis and provides graphical as well as numerical forms of temperature values of the image.

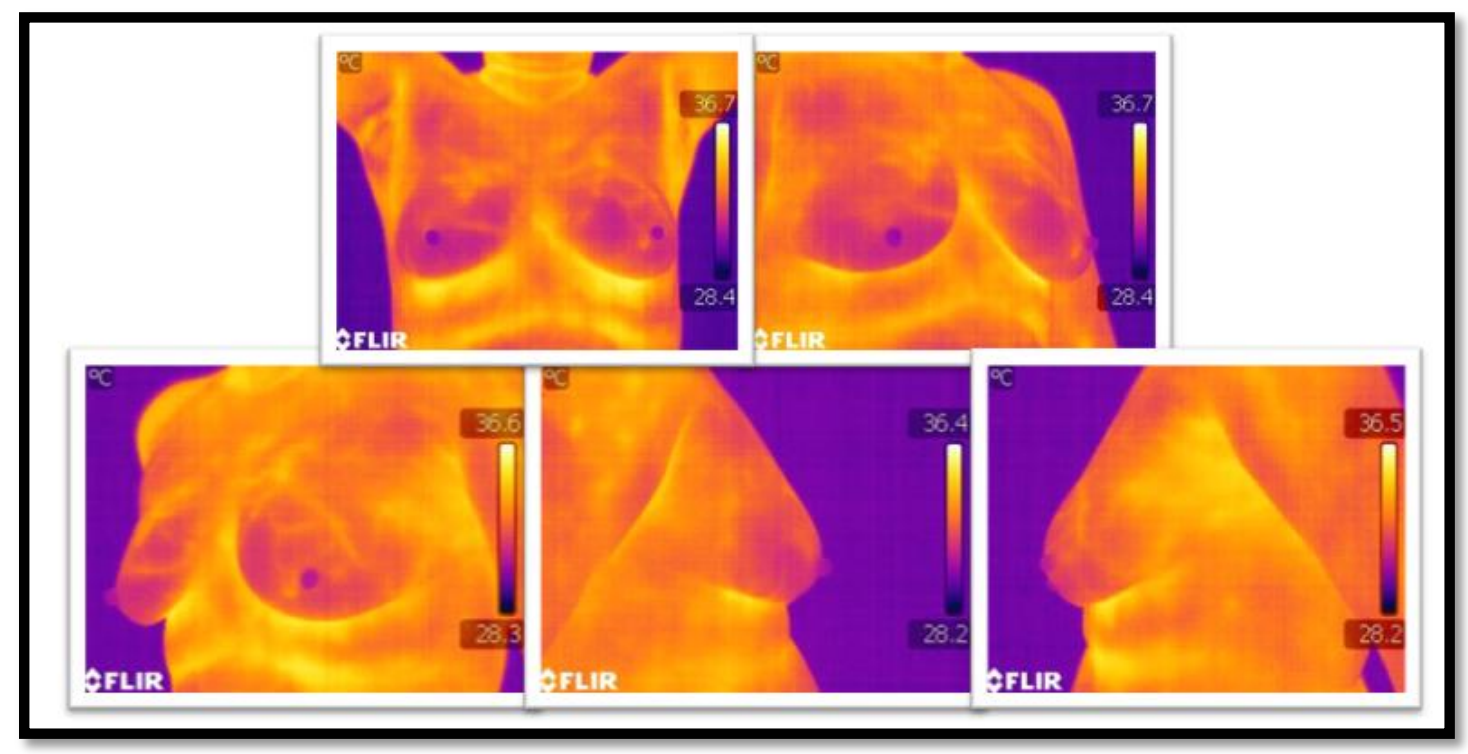

Figure 2: Five Thermal Imaging positions; front, right oblique, left oblique, right \& left sides

\subsection{Image Pre-Processing}

The first step in pre-processing stage intends to use inpaint algorithm for removing the company logo, temperature scale and values in the thermograms. The removal of text from the image is essential to present a clear picture, free of noise for an effective image processing. Inpainting is a process of removing the textual area by filling in with the neighbouring texture [9]. Image inpainting is broadly classified into Texture Synthesis (TS) algorithm and Structure Recreation (SR) [10]. TS is based on the texture outside the region to be inpainted, which usually follows two dimensional pattern with some randomness whereas SR method recreates the structures like lines and object contours which are linear and follow one dimensional pattern. We have considered exemplar based TS method [11] that follows three steps.

1. Computing Filling Priorities; in which a predefined priority function is used to compute the filling order for all the unfilled pixels in the beginning of each filling iteration. 
2. Searching example and compositing; in which the similar texture from the neighboring region is searched to fill the space.

3. Updating image information; in which the filling of the patch area is updated The procedure of inpaint algorithm is shown as flow diagram in Figure 3.

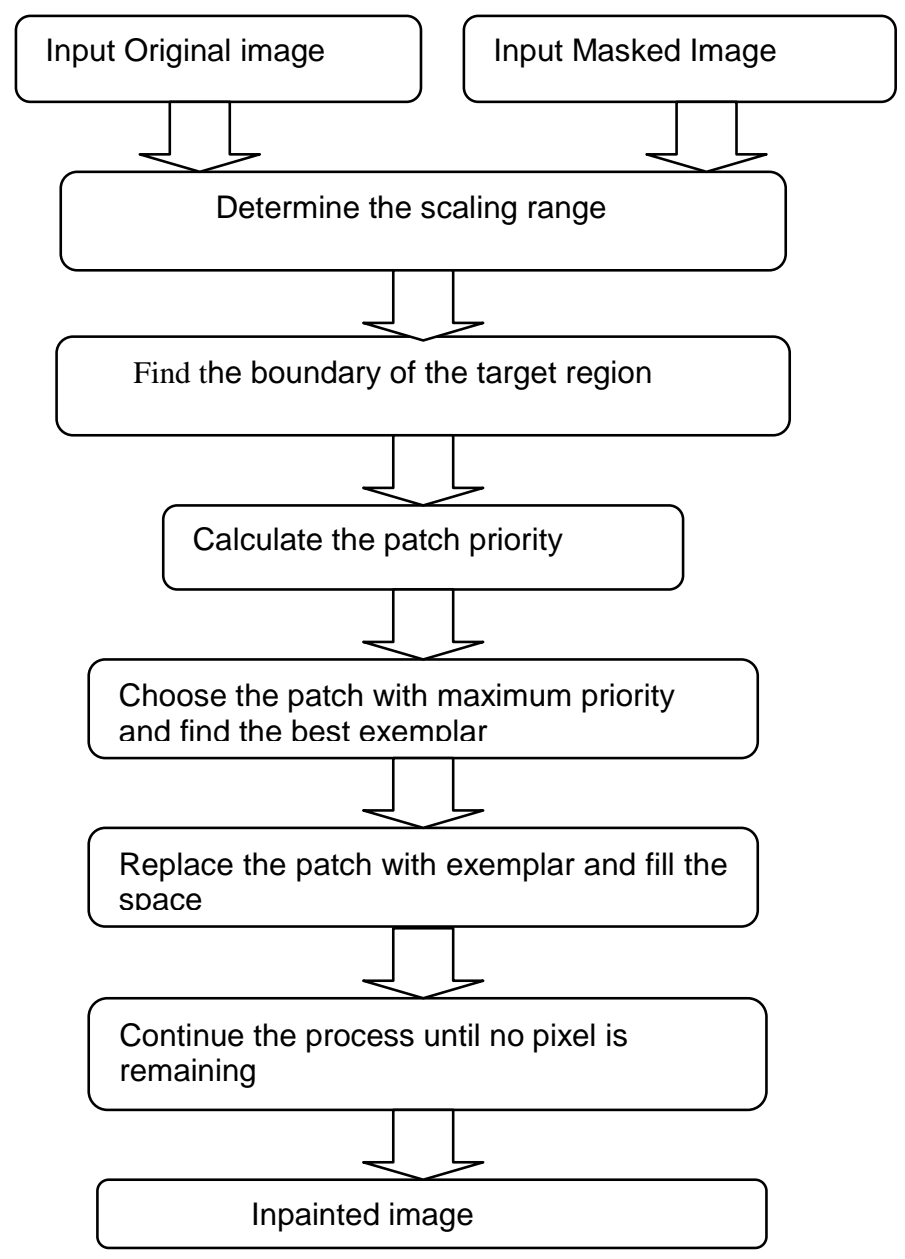

Figure 3: Steps involved in image inpainting

\subsection{Image Enhancement}

Inorder to present the right diagnosis and interpret the thermal breast images, the images must be enhanced for visual quality. The common issue that occurs in medical thermal images is low signal noise ratio and low contrast. The temperature variations inside the breast regions appear as edges and hence, while smoothing the image, these variations which are significant for feature extraction may be blurred and the edges are not preserved.

Recent papers show Partial Differential Equations (PDE) based image processing to be a successful technique due to its advantages in continuous space for shape simplification, structure preserving filtering and enhancement [12]. Traditional filters that are linear, such as Gaussian and median filters perform uniform smoothing operation and hence blur even the important edges [13]. Unlike these filters, non isotropic filter which was first introduced by Perona and Malik is a non linear diffusion process that needs no prior knowledge of the distribution of the noise free image [14]. The raw breast thermal images obtained have unclear and undefined edges which makes the segmentation difficult. Thus the anisotropic diffusion filter is used as smoothing enhancement filter that preserves the sharp edges along the breast boundaries as well as the inter regions of the breast.

The main parameters in isotropic diffusion filtering are conductance function, gradient threshold parameter and stopping time for the iteration. The basic equation of anisotropic diffusion equation proposed by Perona and Malik is given as;

$$
\frac{\partial I(x, y, t)}{\partial t}=\operatorname{div}[g(\|\nabla I(x, y, t)\|) \nabla I(x, y, t)]
$$

where $\mathrm{t}$ is the time parameter, $\mathrm{I}(\mathrm{x}, \mathrm{y}, \mathrm{t})$ is the original image, $\nabla I(x, y, t)$ is the gradient of $\mathrm{I}$ at time $\mathrm{t}$ and $\mathrm{g}()$ is conductance function. For best results in sharper discontinuities $g_{1}$ is proposed by Perona and Malik which favours high 
contrast edges over low contrast ones while $\mathrm{g}_{2}$ is proposed by Black et al [15] which yields sharper edges improving faster diffusion process.

$$
\begin{aligned}
& g_{1}(x)=e^{-\left(\frac{x \sqrt{5}}{s}\right)^{2}} \\
& g_{2}(x)=\left\{\begin{array}{cc}
0.67\left[1-\left(\frac{x}{S}\right)^{2}\right]^{2} ; x \leq S \\
0 & ; \text { Otherwise }
\end{array}\right.
\end{aligned}
$$

where, $\mathrm{S}=\mathrm{k} \sqrt{2} \cdot \mathrm{k}$ is gradient threshold parameter that controls the rate of the diffusion. The anisotropic diffusion filtering has a good edge preserving behavior but it is incapable of smoothing high level noise efficiently. Thus the choice of gradient threshold parameter play an important role as it defines the threshold between image gradients that are attributed to noise and those attributed to true edges. The threshold parameter $(\mathrm{K})$ can be estimated by applying Gaussian filter of scale $\sigma$ and the gradient is computed using smoothed version of the image $g\left(\left\|\nabla\left(G_{\sigma} * I(x, y, t)\right)\right\|\right)$ in every iteration [16]. The discrete anisotropic diffusion equation is given as;

$$
I_{t+1}(s)=I_{t}(s)+\frac{\lambda}{\left|\eta_{s}\right|}\left[\sum_{p \in N, S} g\left(\nabla I_{s, p}\right)+\sum_{p \in E, W} g\left(\nabla I_{s, p}\right)\right]
$$

The choice of the stopping time T needs to be careful as it could lead to either blurring the true edges or leave the noise unfiltered. Hence, the proposed scheme of [17] has been used for stopping time $T$ in this paper.

\subsection{Image Segmentation}

The application of image segmentation technique depends on the type of the image and its requirement. For this reason, regions in the images are characterized. Segmentation is essential for the breast thermal images to extract the region of interest i.e. both left and right breasts for better medical interpretation. Level set method is used for the segmentation in this paper. The level set evolution method follows two traditional steps: edge detection, to find relevant boundaries and region growing to reach the whole region [18]. Such type of segmentation technique falls under active contours which were introduced by Kass, Witkin and Terzopoulous. Active contours are broadly classified as either parametric or geometric active contour model [19]. The level set function is used to represent the zero level of the contours and evolution takes place by using partial differential equation (PDE). This is also known as variational level set method (Sethian et al., 1999). The traditional evolution equation of level set function is given by

$$
\frac{\partial \phi}{\partial t}+F|\nabla \phi|=0
$$

$F$ is the speed function. During the evolution, it is necessary to keep the level set function close to signed distance function due to sharper shapes hence, LSF $\phi$ needs to be re-initialized and reshaped as signed distance function

$$
\frac{\partial \phi}{\partial t}=\operatorname{sign}\left(\phi_{0}\right)(1-|\nabla \phi|)
$$

$\phi_{0}$ is the function to be re-initialized and $\operatorname{sign}(\phi)$ is the signed function.

This paper has adapted variational formulation without using re-initialization [20] which incorporates internal energy term and an external energy term. The internal energy maintains the closeness between the level set function and signed distance function whereas, the external energy drives the initial contour towards the object boundaries. Hence, the total variational formulation is given by

$$
\varepsilon(\phi)=\mu P(\phi)+\varepsilon_{m}(\phi)
$$
defined as

where, $\mu$ is a positive constant and $\mu P(\phi)$ is the internal energy that maintains the signed distance function [21]

$$
P(\phi)=\frac{1}{2} \int_{\Omega}(|\nabla \phi|-1)^{2} d x d y
$$

The external energy that drives the zero level set to the boundaries is given as [22]

$$
\varepsilon_{g, \lambda, v}(\phi)=\lambda L_{g}(\phi)+v \mathrm{~A}_{g}(\phi)
$$

$\lambda$ and $v$ are positive constants, $L_{g}(\phi)$ and $\mathrm{A}_{g}(\phi)$ are defined as

$$
L_{g}(\phi)=\int_{\Omega} g \delta(\phi)|\nabla \phi| d x d y
$$




$$
A_{g}(\phi)=\int_{\Omega} g H(-\phi) d x d y
$$

where $\delta$ is the univariate Dirac function and $\mathrm{H}$ is the Heaviside function. $\mathrm{g}$ is used as edge indicator for the level set function to evolve towards the boundaries and defined as

$$
\begin{aligned}
& g=\frac{1}{1+\left|\nabla G_{\sigma} * I\right|^{2}} \\
& g=\frac{1}{1+\nabla\left|I_{t}\right|^{2}}
\end{aligned}
$$

if $I$ is the original image, $G_{\sigma}$ is the Gaussian kernel with standard deviation $\sigma$ and $I_{t}$ is the filtered image using anisotropic diffusion filter. $\frac{\partial \varepsilon}{\partial \phi}$ is the first variation hence, $\frac{\partial \phi}{\partial t}=-\frac{\partial \varepsilon}{\partial \phi}$ is the gradient flow. By calculus of variation, the Gateaux derivative of the functional $\varepsilon$ is written as

$$
\frac{\partial \varepsilon}{\partial \phi}=-\mu\left[\Delta \phi-\operatorname{div} \frac{\nabla \phi}{|\nabla \phi|}\right]-\lambda \delta(\phi) \operatorname{div}\left(g \frac{\nabla \phi}{|\nabla \phi|}\right)-v g \delta(\phi)
$$

The gradient flow equation that minimizes the energy function $\varepsilon$ is given as

$$
\frac{\partial \phi}{\partial t}=\mu\left[\Delta \phi-\operatorname{div} \frac{\nabla \phi}{|\nabla \phi|}\right]+\lambda \delta(\phi) \operatorname{div}\left(g \frac{\nabla \phi}{|\nabla \phi|}\right)+v g \delta(\phi)
$$

\section{Experimental Results}

Original thermal images of normal breast, lactating breast and fibroadenoma breast in five positions are shown in Figures (4-6). It is seen that normal breast thermogram has symmetrical temperature variations in both the breasts. The lactating breast shows high vascular regions which is distributed evenly around both the breasts. Due to active milk ducts, the thermogram appears to be highly vascularised. The fibroadenoma breast image clearly shows an asymmetric pattern on the right breast when compared to its contralateral part.

The pre-processing step is presented in Figure (4-6 (f-j)). Inpaint algorithm was applied to the original images to remove the text, temperature values and labels. Figure 7 shows the mask used in the inpaint algorithm. The images are then subjected to both Gaussian and anisotropic diffusion filters.

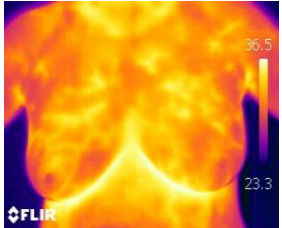

(a)

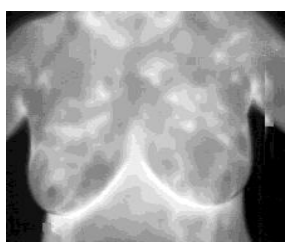

(f)

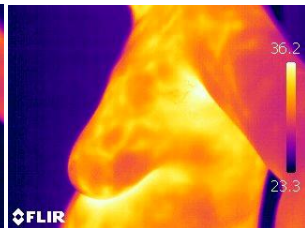

(b)

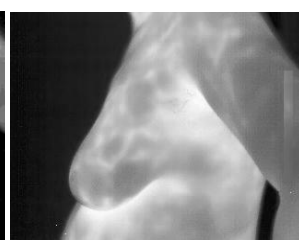

(g)

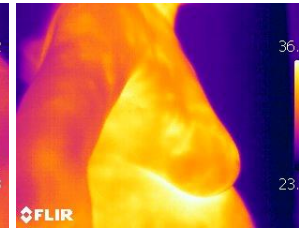

(c)

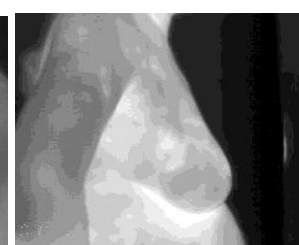

(h)

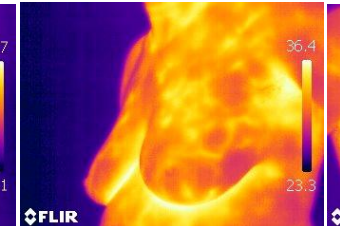

(d)

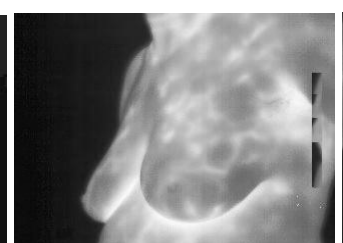

(i)

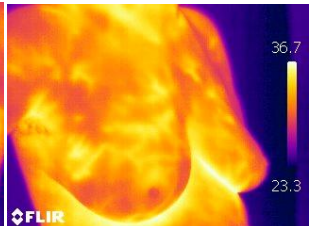

(e)

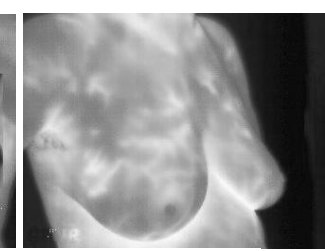

Figure 4. Original image of normal breast in five imaging positions and results of label removing using image inpaint $(a-e)$ front view, left side, right side, left lateral and right lateral ( $f-j)$ results of label removing

Figures (8-10) shows the corresponding edge maps. On using Gaussian filter with sigma values 2 and more, the entire image is smoothened and blurred. The edge map obtained by Gaussian kernel contains noise and edges are not preserved. Hence, anisotropic diffusion filter is adapted inorder to preserve the edges and filter the noise by smoothing the images.

The main parameters for anisotropic filter are $\mathrm{K}$ and $\mathrm{T}$. They are optimized based on equation 2 and 3 . The optimal value of $\mathrm{K}$ was chosen to be 11 for normal breast and 8 for the lactating and fibroadenoma breast images. These values were chosen such that it smoothes inner regions of the breast and preserves the higher gradient areas such as breast boundaries. 


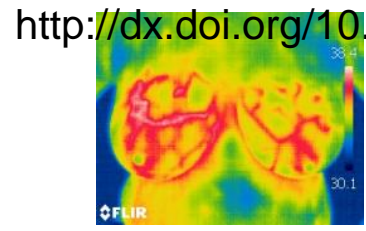

(a)

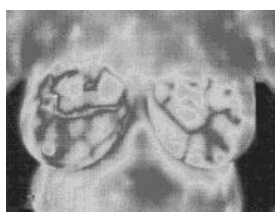

(f)

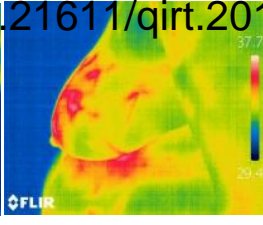

(b)

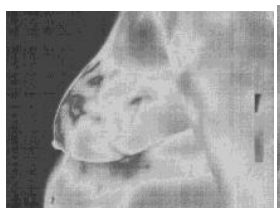

(g)
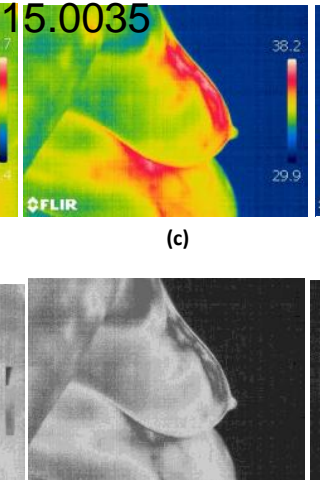

(h)

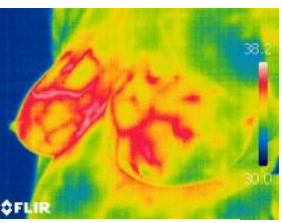

(d)

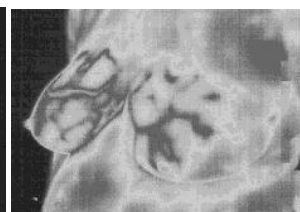

(i)

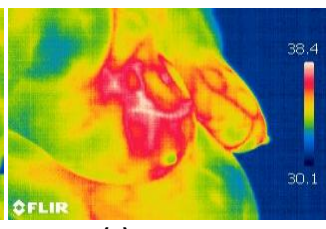

(e)

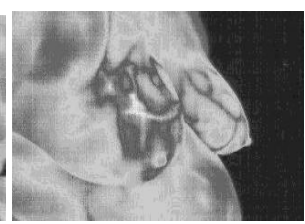

(j)

Figure 5. Original image of lactating breast in five imaging positions and results of label removing using image inpaint (a-e) front view, left side, right side, left lateral and right lateral (f-j) results of label removing

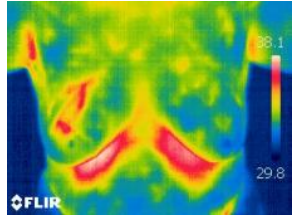

(a)

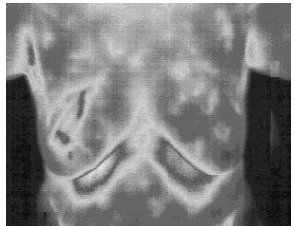

(f)

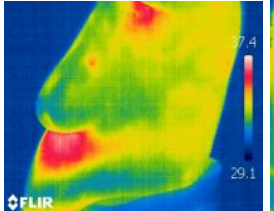

(b)

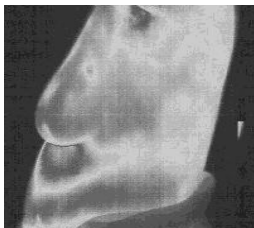

(g)

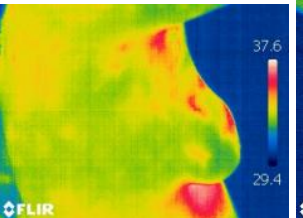

(c)

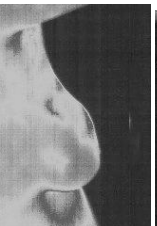

(h)

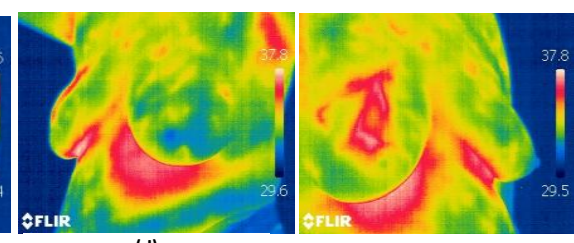

(e)

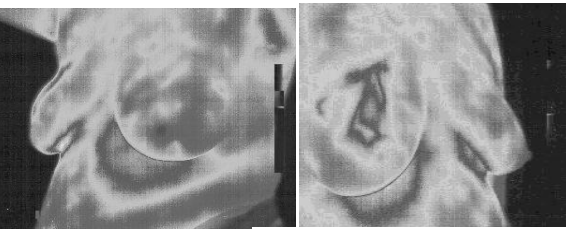

(j)

Figure 6. Original image of fibro adenoma breast in five imaging positions and results of label removing using image inpaint (a-e) front view, left side, right side, left lateral and right lateral (f-j) results of label removing

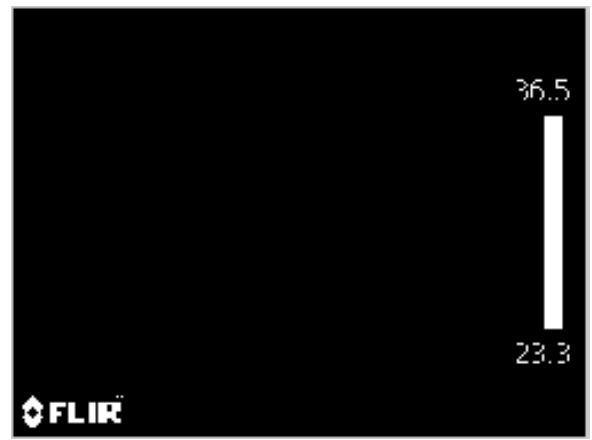

Figure 7: Mask used in Inpaint algorithm

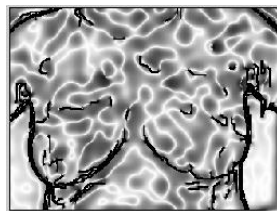

(a)

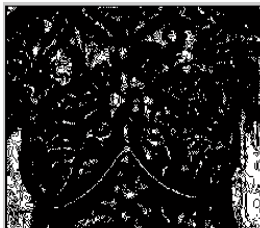

(f)

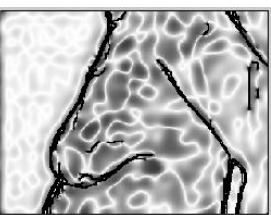

(b)

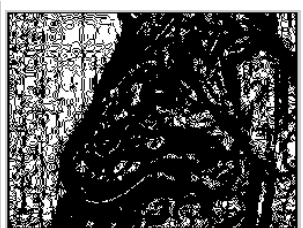

(g)

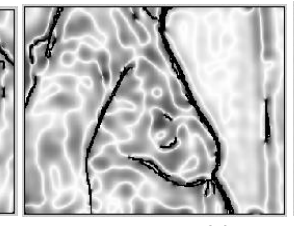

(c)

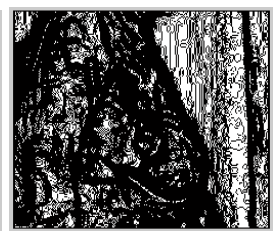

(h)

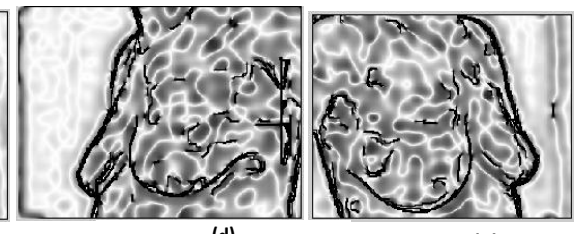

(e)

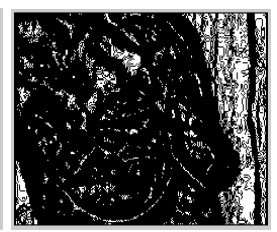

(j)

Figure 8. Edge map results of normal breast in five imaging positions (a-e) edge map using anisotropic diffusion filter, $(f-j)$ edge map using Gaussian filter 


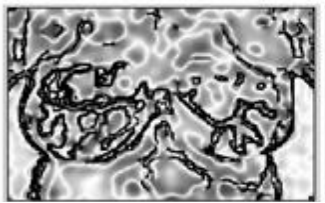

(a)

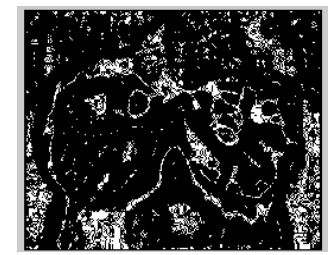

(f)

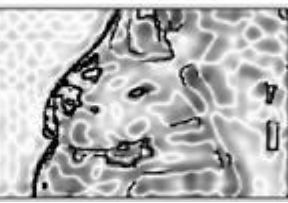

(b)

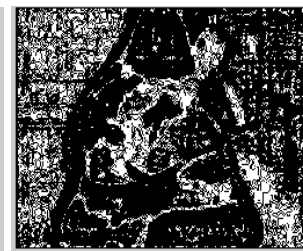

(g)

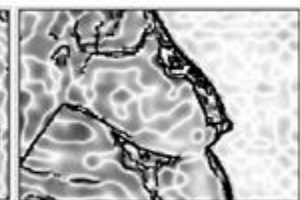

(c)

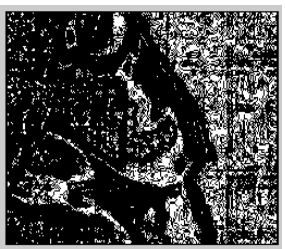

(h)

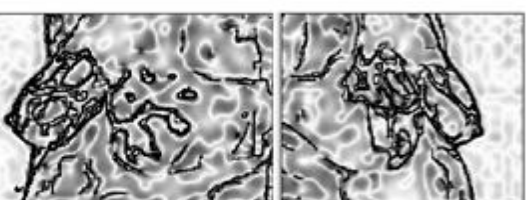

(d)

(e)

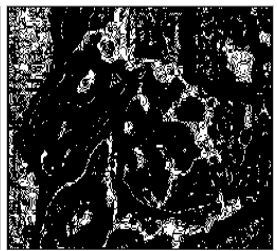

(i)

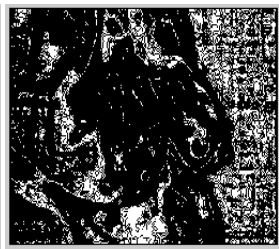

(j)

Figure 9. Edge map results of lactating breast in five imaging positions (a-e) edge map using anisotropic diffusion filter, (f-j) edge map using Gaussian filter

The breast boundaries are preserved and the inner regions are smoothed inorder to correctly segment the area of interest using level set evolution. The initial contour is set according to the equation given below

$$
\Omega_{0}(x, y)=\left\{\begin{array}{rr}
-\rho, & (x, y) \epsilon \Omega_{0}-\delta \Omega_{0} \\
0, & (x, y) \epsilon \delta \Omega_{0} \\
\rho, & \Omega-\Omega_{0}
\end{array}\right.
$$

where $\rho>0$ is a constant, $\rho$ is chosen larger such that the body is covered within the bounding box. The final segmented regions for the three breast types in all imaging positions are given in Figure 11. The segmented images present the region of interest by which further image processing techniques can be applied to analyze the breast tissue whether it is normal or abnormal.

\section{Performance Evaluation}

The segmented result is compared with the reference images that are manually segmented by radiologists and experts. The measures used to evaluate the segmentation performance are Jaccard Index (JC), Dice Similarity (DS), Tanimoto (TN), Structural Similarity (SSIM) and Volume Similarity (VS) which are given below [23],

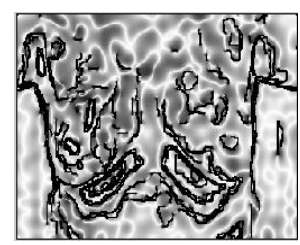

(a)

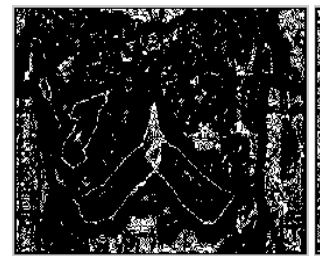

(f)

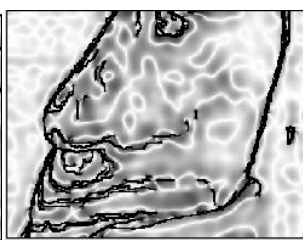

(b)

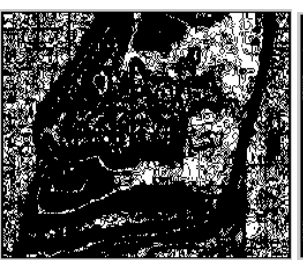

(g)

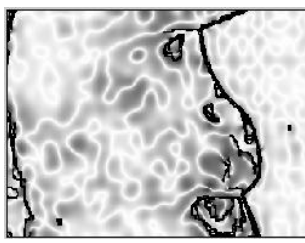

(c)

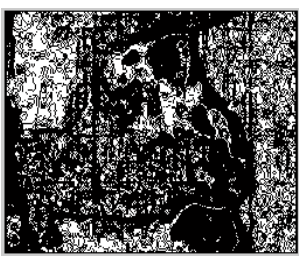

(h)

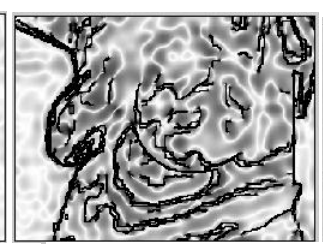

(d)

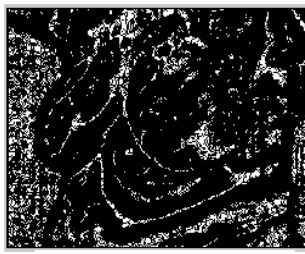

(i)

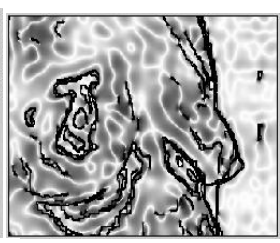

(e)

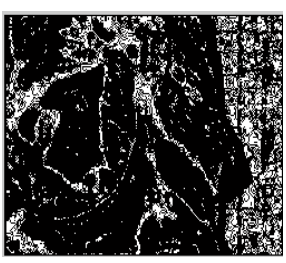

(j)

Figure 10. Edge map results of fibro adenoma breast in five imaging positions (a-e) edge map using anisotropic diffusion filter, $(f-j)$ edge map using Gaussian filter 
http://dx.doi.org/10.21611/qirt.2015.0035

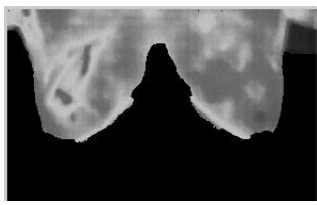

(i)

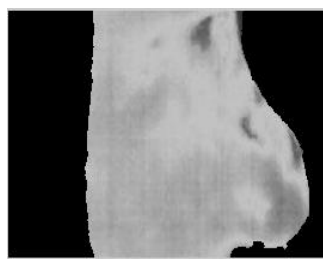

(ii)

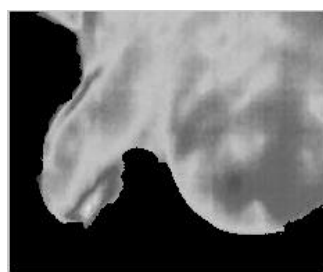

(iii)

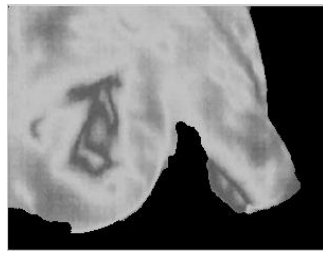

(iv)

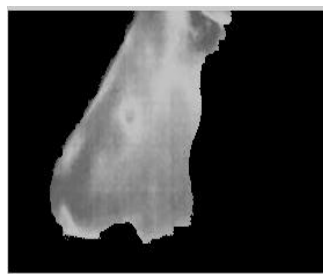

(v)

(a)

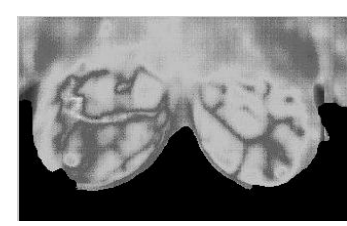

(i)

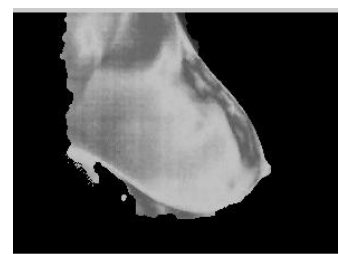

(ii)

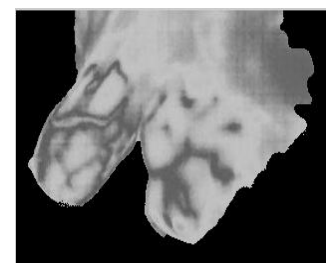

(iii)

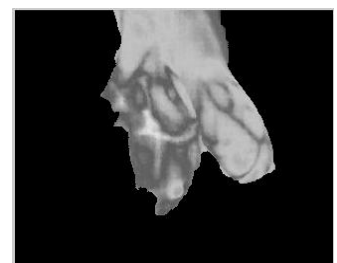

(iv)

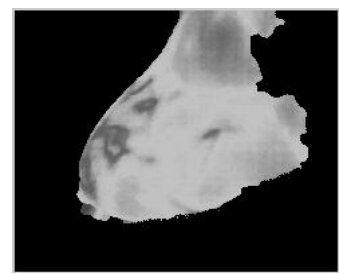

(v)

(b)

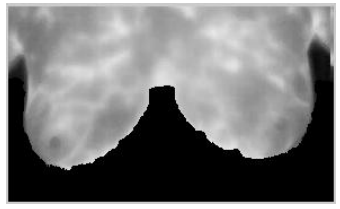

(i)

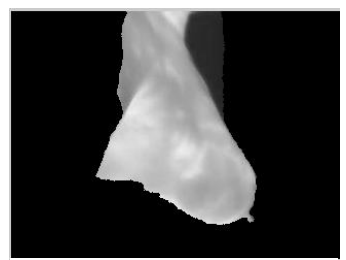

(ii)

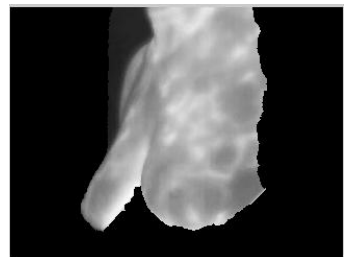

(iii)

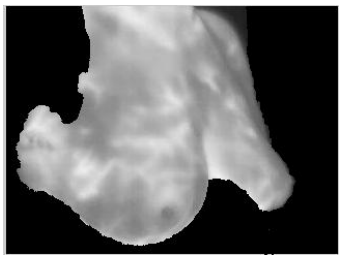

(iv)

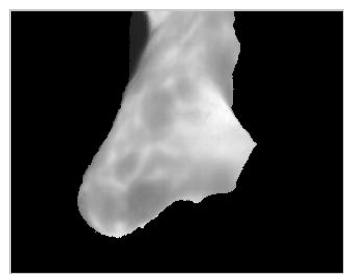

(v)

(c)

Figure 11: Segmentation results of $(a, i-v)$ Fibroadenoma Breast $(b, i-v)$ Lactating breast and (c,i-v)Normal Breast

$$
\begin{aligned}
& \mathrm{JC}=\frac{|A \cap B|}{|A \cup B|} \\
& \mathrm{DS}=\frac{2|A \cap B|}{|A|+|B|}
\end{aligned}
$$

The above two coefficients are equal to 1 if $A$ and $B$ are the same regions, and 0 if they are disjoint regions, $A$ is the segmented image and $B$ is the reference image.

$$
\begin{aligned}
& \mathrm{TN}=\frac{|A \cap B|+\overline{|A \cup B|}}{|A \cup B|+\overline{|A \cap B|}} \\
& \mathrm{VS}=1-\frac{|| A|+| B||}{|A|+|B|}
\end{aligned}
$$

TN is 1 if $A$ is equal to $B$ and 0 if they are disjoint regions and they occupy the entire image. VS is 1 if the number of elements of $A$ is equal to the number of elements of $B$ and 0 if one of the images is empty. Figures (12-14) show the bar plot presentation of segmentation measures between the reference image and segmented image for normal, lactating and fibroadenoma breast images in all five imaging positions. The DC and TN give 99\% similarity 
showing maximum overlapping regions between segmented and reference images. Other measures also show an average of $98 \%$ of similarity.

\section{Patient 1 - Normal Breast}

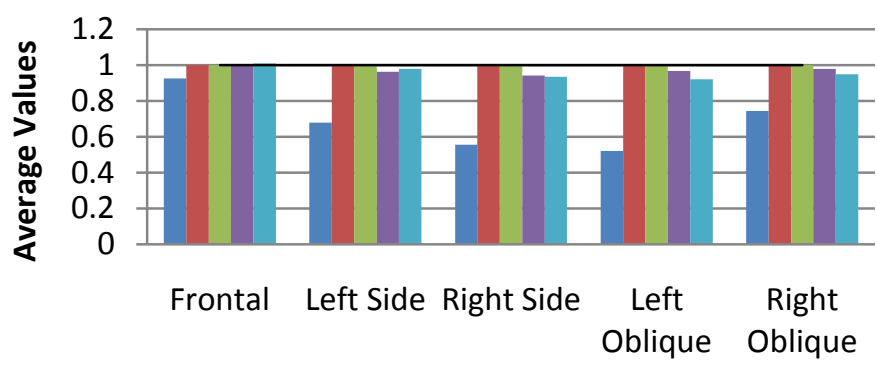

Jaccard Index

Dice Coefficient

Tanimoto

SSIM

Breast Positions

Volume Similarity

Linear (Dice Coefficient)

Figure 12: Bar plot presentation of overlap measures obtained between segmented images and reference images in all five imaging positions for the normal breast

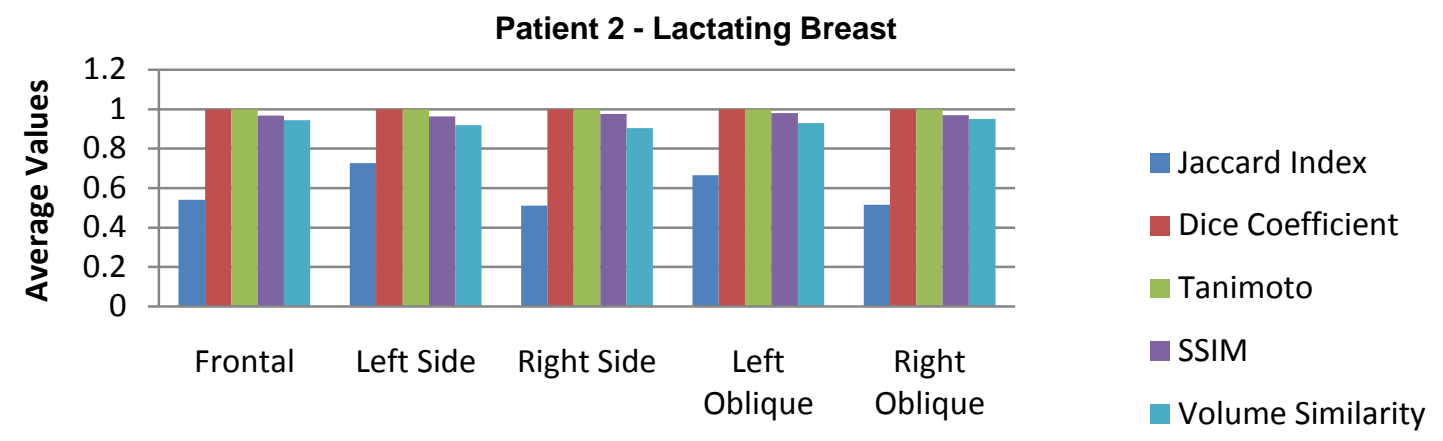

Breast Positions

Figure 13: Bar plot presentation of overlap measures obtained between segmented images and reference images in all five imaging positions for the lactating breast

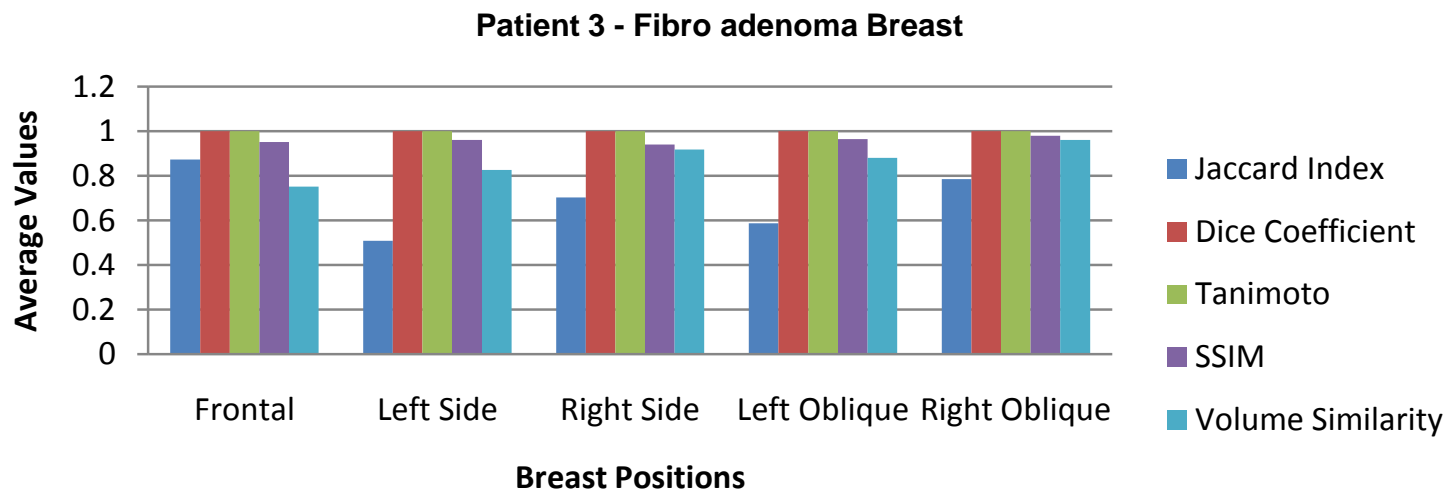

Figure 14: Bar plot presentation of overlap measures obtained between segmented images and reference images in all five imaging positions for Fibro adenoma breast 


\section{Discussion and Conclusion}

Segmentation of desired region from the breast thermogram plays an essential role in the diagnosis. Thermograms continue to be degraded until proper protocols are formulated for imaging the patient. Lower breast boundaries and infra mammary fold below the breast line pose many difficulties while segmenting the region of interest. In this paper, we have carried out pre-processing steps such as label and text removal using inpaint algorithm. The anisotropic diffusion filter produced clear cut edge maps with distinguished edges and breast boundaries which gave satisfactory segmentation results using level set evolution method. The level set without reinitialization technique has the potential in accurately extracting the breast ROI without any manual intervention for selecting the contour. The five imaging positions give relevant information while the radiologists interpret and analyze the breasts; hence the segmentation process was employed for all the five positions too. The performance was evaluated by comparing the obtained results with the reference images. The graph plot presentations show that the evolution method can be used for segmentation of ROI in breast thermogram.

\section{REFERENCES}

[1] International Agency for Research on Cancer Available at http://www.iarc.fr. [Accessed Jan 2014].

[2] Gautherie M, Gros C, Contribution to infrared thermography to early diagnosis, pre therapeutic prognosis and post irradiation follow up of breast carcinomas. Med. Mundi 1976; 21:135-149.

[3] Gros C, Gautherie M, Bourjat P, Prognosis and post therapeutic follow up of breast cancers by thermography. Bibl. Radiol; 6:77-90; 1975

[4] Lawson RN, Chughtai MS, Breast cancer and body temperatures. Can Med Assoc J ; 88:68-70, 1963.

[5] Anbar M, Clinical thermal imaging today. IEEE Eng Med Biol Mag, ; 17: 25-33,1998.

[6] N. G. E. Y, Kee E C Advanced integrated technique in breast cancer thermography. J Med Eng Technol, ; 32:103:14,2008.

[7] Antonini S, D, Kolaric I.A, Nola Z, Herceg, V. Ramljak, T. Kulis, J.K Holjevek and Z. Ferencic, Thermography surveillance after breast conserving surgery-three cases, $53^{\text {rd }}$ International Symposium ELMAR, Croatia, September, ; 317-319, 14-16; 2011.

[8] Keyserlingk J R, Ahlgren P D, YU E, Belliveau N, Yassa M., Functional infrared imaging of the breast - Historical perspectives, current applications and future considerations, IEEE Eng Med Biol, 19:30-41. 2000.

[9] Bertalmio M, Casseler V, Ballester C, Sapiro G, Image Inpainting, ACM Press, 2000.

[10] Chen Q, Zhang Y, Liu Y, Image Inpainting with improved exemplar based approach, Multimedia Content Analysis and Mining, LNCS, vol. 4577/2007, 242-251, 2007.

[11] Criminisi A, Perez P, Toyamma K, Region filling and object removal by exemplar based image inpainting, IEEE Trans on Image Proc, 13(9), 1200-1212, 2004.

[12] Weichert J, Anisotropic Diffusion in Image Processing, vol 1, Teubner, Stuttgart, 1998.

[13] Chao SM, Tsai DM, An improved anisotropic diffusion model for detailed and edge preserving smoothing, Pattern Recognition Letters 31(13), 2012-2023, 2010.

[14] Perona P, Malik J, Scale -space and edge detection using anisotropic diffusion, IEEE Trans on Pattern Analysis and Machine Intelligence, 12 (7), 629-639, 1990.

[15] Black MJ, Sapiro G, Marimont D, Heeger D, Robust anisotropic diffusion, IEEE Trans on Image Processing 7(3), 421-432, 1998.

[16] Tsiotsios C, Petrou M, On the choice of the parameters for anisotropic diffusion in image processing, Pattern Recognition, 1-13, 2012.

[17] Mitiche A, Ayed I B, Variational and Level Set Methods in Image Segmentation. Benesly J, Canada and Kellesmann W, Germany, editors. Springer Topics in Signal Proceesing, Springer, vol. 5, 2010.

[18] Kass M, Witkin A, Terzopoulous D, Snakes: Active Contours models, Int J. Computer Vision, vol. 1, 321-331, 1987.

[19] Li C, Xu C, Gui C, Fox D, Level set evolution without re-initialization: A new variational formulation, in : IEEE Computer Society conference on Computer Vision and Pattern Recognition, vol. 1, 430-436, 2005.

[20] Xu C, Prince J, Snakes, shapes and gradient vector flow, IEEE Trans Imag. Proc., vol 7, 359-369, 1998.

[21] Cardemes R, Rd L. G, Cudra M. B, A Multidimensional Segmentation Evaluation for Medical Image Data, Computer Methods and Programs in Biomedicine 96, 108-124; 2009.

[22] Arnold V I, Geometrical methods in the theory of ordinary differential equations, Springer-Verlag, New York, 1983.

[23] Sethian J A, Level set methods and fast marching methods, Cambridge: Cambridge University Press, 1999. 